\title{
Methyl-CpG binding protein MBD2 is implicated in methylation- mediated suppression of miR-373 in hilar cholangiocarcinoma
}

\author{
YONGJUN CHEN ${ }^{1}$, WEI GAO ${ }^{2}$, JIAN LUO $^{1}$, RUI TIAN $^{1}$, HUAWEN SUN $^{3}$ and SHENGQUAN ZOU ${ }^{1}$
}

\author{
Departments of ${ }^{1}$ Biliary-Pancreatic Surgery, and ${ }^{2}$ Traumatology, Tongji Hospital, Tongji Medical College, \\ Huazhong University of Science and Technology 1095, Jiefang Road, Wuhan 430030; ${ }^{3}$ Department of Gastrointestinal \\ Surgery, Renmin Hospital of Wuhan University 238, Jiefang Road, Wuchang, Wuhan 430060, P.R. China
}

Received August 31,2010; Accepted October 19, 2010

DOI: $10.3892 /$ or.2010.1089

\begin{abstract}
Aberrant expression of miRNAs is associated with particular cancers showing tissue- and clinical-featurespecificity patterns. Some miRNA genes harboring or being embedded in $\mathrm{CpG}$ islands undergo methylation mediated silencing. MBP, methyl CpG binding protein, suppresses transcription through binding to methylated $\mathrm{CpG}$ dinucleotides. Expression of miR-373 has been reported to be suppressed in malignant bile duct cell lines. Bioinformatic prediction reveals that the transcription start site (TSS) of miR-373 is implanted in a 402 bp canonical $\mathrm{CpG}$ island containing $26 \mathrm{CpG}$ dinucleotides. In this study, we aim to determine the epigenetic regulation of miR-373 gene in hilar cholangiocarcinoma. TaqMan microRNA assay shows that downregulation of miR-373 is closely associated with poor cell differentiation, advanced clinical stage and shorter overall and disease-free survival in hilar cholangiocarcinomas. Methylation analysis shows that the promoter-associated $\mathrm{CpG}$ island is hypermethylated which is consistent with the inhibition of miR-373. Chromatin immunoprecipitation (ChIP) assay indicates that down-regulation of miR-373 resultes from the selective recruitment of MBD2 to methylated $\mathrm{CpG}$ islands. In contrast, MBD2 knock-down by use of a specific siRNA promoted the expression of miR-373. Reactivation of miR-373 by pharmacologic induction of 5aza-CdR and trichostatin A (TSA) led to decreased enrichment of MBD2 at $\mathrm{CpG}$ island regions. Enhanced expression of exogenous MBD2 in stable $\mathrm{QBC}_{939}$ cells which stably express pGL4-m373-prom induces strengthened recruitment of MBD2. Our findings suggest that miR-373 is a methylationmediated gene and the implication of MBD2 in methylationmediated suppression of miR-373 plays an important role
\end{abstract}

Correspondence to: Dr Yongjun Chen, Department of BiliaryPancreatic Surgery, Tongji Hospital, Tongji Medical College, Huazhong University of Science and Technology 1095, Jiefang Road, Wuhan 430030, P.R. China

E-mail: zigachen45@gmail.com

Key words: MBD2, miR-373, DNA methylation, hilar cholangiocarcinoma in tumourigenesis and development in hilar cholangiocarcinoma.

\section{Introduction}

Hilar cholangiocarcinoma, known as Klatskin tumor (1), is an uncommon cancer with an incidence of 0.01-0.2\% per year (2). Although it is relatively rare, hilar cholangiocarcinoma displays highly aggressive malignancy and is considered to be an incurable and rapidly lethal disease despite recent progresses in diagnostic and therapeutic techniques. Five-year overall survival after curative resection ranges from 20 to $40 \%$ and 10 -year survival is almost zero $(3,4)$. Furthermore, patients with inoperable, recurrent, or metastatic disease can only be treated with palliative therapy, such as endoscopic, percutaneous biliary drainage (5) combination with radiotherapy and chemotherapy. However, hilar cholangiocarcinoma is not sensitive to chemotherapy and radiotherapy $(6,7)$, the median survival rate of those cases is around 9-12 months only (8).

Aberrant DNA methylation has been validated as an essential mechanism in regulation of gene expression in human cancer (9). Once a given sequence becomes methylated, it either can repress transcription directly by impeding recognition of transcriptional activators to DNA sequences (10); or by binding methyl-CpG binding domain proteins (MBPs) to modify chromatin compaction and control gene silencing (11). For the reason that not all transcription factor recognition sequences contain $\mathrm{CpG}$ dinucleotides, the second model is considered as primary pathway in methylation-mediated silencing (12).

Biochemical and genetic analysis of MBPs have established direct link between DNA methylation and repressive chromatin architecture (13). The involvement of MBPs in gene imprinting (14), X inactivation (15), and transcriptional silencing of genes possessing hypermethylated $\mathrm{CpG}$ islands in cancer cells (16) is now well documented. MBPs includes 5 isoforms of Mecp2, MBD1, MBD2, MBD3, and MBD4. With the exception of MBD4, which is primarily a thymine glycosylase involved in DNA repair (17), all MBPs are implicated in the transcriptional repression mediated by DNA methylation. Several lines of evidence have demonstrated that Mecp2, MBD1 and MBD2 are involved in methylation-based gene repression and also affect chromatin 
structure (18-20). MBD3 lacks functional methylation binding domain (MBD) but is an integral subunit of Mi2-NuRD complex which is recruited through MBD2 (21).

microRNAs (miRNAs) have recently been suggested to play important roles in cancers as $>50 \%$ of miRNA genes reside in cancer-associated genomic regions and their expressions have been found to be dysregulated in various cancers (22). Increasing evidence has shown profound impact of miRNAs on many processes that are frequently disrupted during cell proliferation, apoptosis, and differentiation (23). Depending on the target tumor-relative genes, miRNAs behave as tumor suppressor genes or oncogenes $(24,25)$. Importantly, with the expression pattern of strict specificity of tissue and clinical feature, differential express of miRNAs could be used as diagnostic and/or prognostic markers for particular cancer.

Mature miRNAs are transcribed from miRNA gene by RNA pol II (26), so it is believed that the transcription of miRNA genes share the same genetic and epigenetic regulation with functional gene including promoter methylation (27). Although only subsets of miRNA genes harbor $\mathrm{CpG}$ island in promoter region or are embeded in $\mathrm{CpG}$ island, evidence on DNA methylation-mediated regulation of miRNAs by proximal $\mathrm{CpG}$ islands have been mounted in number of groups.

In this study, we demonstrate miR-373, a reported specific microRNA to malignant bile duct cell lines, is inhibited by selective recruitment of MBD2 at the hypermethylated promoter-associated $\mathrm{CpG}$ island in hilar cholangiocarcinoma.

\section{Materials and methods}

Patients and cell lines. In total 48 patients (Table I) with both tumor and normal bile duct tissues obtained from operation successfully were included into this study at Tongji Hospital, Tongji Medical College, Huazhong University of Science and Technology (China) from January 2005 to December 2008. Written informed consent was obtained from patients before sample collection. The study was approved by the Review Board of Tongji Medical College and Hospital.

Cell lines and epigenetic treatment. $\mathrm{QBC}_{939}$ cell line originated from human common bile duct adenocarcinoma was kindly provided by Dr W. Shuguang from Southwest Hospital of the Third Military Medical University (China) (28). HEK293 cell were purchased from Cell bank of Chinese Academy of Sciences. For epigenetic study, $\mathrm{QBC}_{939}$ cells were treated with $5.0 \mu \mathrm{M} 5$-aza-CdR for 5 days and $200 \mathrm{nM}$ of trichostatin A (TSA; Sigma-Aldrich) was added at day 5 (29).

TaqMan microRNA assay. RNAs were extracted using mirVana $^{\mathrm{TM}}$ miRNA Isolation Kit (Applied Biosystems). cDNA synthesis and expression of miR-373 were performed with TaqMan MicroRNA Assay (ABi). U6 (RUN6B) was assessed as endogenous control. Expression of miR-373 was normalized to U6 and fold change was calculated based on the $2^{-\Delta \Delta C t}$ method (30).

Genome DNA bisulfate modification. Genome DNA was isolated from tissues and cells using the DNeasy ${ }^{\circledR}$ Blood \&
Table I. Relationship between miR-373 expression and clinicopathological features.

\begin{tabular}{lccc}
\hline $\begin{array}{l}\text { Clinicopathological } \\
\text { features }\end{array}$ & $\begin{array}{c}\text { No. of } \\
\text { patient }^{\mathrm{a}}\end{array}$ & $\begin{array}{c}\Delta \mathrm{C}_{\mathrm{t}} \text { value } \\
\text { of miR-373 }\end{array}$ & P-value \\
\hline
\end{tabular}

Age (years)
$<60$
$\geq 60$

$19 \quad 27.69 \pm 3.76 \quad 0.059$

$\geq 60$

$29 \quad 25.65 \pm 4.35$

Gender

Male

Female

$33 \quad 24.43 \pm 2.43$

$15 \quad 29.01 \pm 3.76$

Tumor size $(\mathrm{cm})$

$\begin{array}{llll}<2 & 29 & 25.92 \pm 3.64 & 0.606 \\ \geq 2 & 31 & 25.49 \pm 2.59 & \end{array}$

Pathological type

Adenocarcinoma

Mucocellulare carcin

44

$26.71 \pm 3.18$

0.39

Adenosquamous carcinoma

$24.63 \pm 3.57$

Squamous carcinoma

23.94

Undifferentiated carcinoma

27.91

Cell differentiation

$\begin{array}{lll}\text { Well } & 14 & 19.09 \pm 3.46 \\ \text { Moderately } & 23 & 21.97 \pm 1.74 \\ \text { Poorly } & 11 & 28.43 \pm 4.09\end{array}$

$0.031^{\mathrm{a}}$

Poorly

$28.43 \pm 4.09$

Bismuth classification

I

II

III

$24.66 \pm 3.31$

0.082

IV

$27.65 \pm 2.71$

$26.99 \pm 4.02$

$26.22 \pm 3.96$

Lymphatic node metastasis

Absent

$19 \quad 28.59 \pm 2.53$

Present

$29 \quad 25.01 \pm 2.64$

0.224

Clinical stages

$\begin{array}{lll}\text { I + II } & 13 & 18.35 \pm 2.62 \\ \text { III + IV } & 35 & 27.95 \pm 3.12\end{array}$

$0.017^{\mathrm{a}}$

Data are presented as the means \pm SEM and were analyzed using the Student's t-test or one-way ANOVA depending on data characteristics. ${ }^{\mathrm{a}} \mathrm{P}<0.05$.

Tissue Kit (Qiagen). For DNA methylation detection, $1.5 \mu \mathrm{g}$ of genomic DNA was modified with sodium bisulfite using the EpiTect Bisulfate Kit (Qiagen). CG Genome Universal Unmethylated DNA (uDNA) and CG Genome Universal Methylated DNA (mDNA) (Millipore) were also modified for positive and negative controls (100\% values).

DNA methylation analysis. Methylation specific PCR (MSP) and MethySYBR $(31,32)$ qMSP were performed with primers specific for fully methylated and fully unmethylated $\mathrm{CpG}$ island sequences (MmiR-373, UmiR-373). Primers for converted (ActB) and unconverted (ActG) B-actin special sequence containing no $\mathrm{CpG}$ sites were used as control to correct $\mathrm{C}_{\mathrm{T}}$ values and efficiency of bisulfite conversion, 
respectively. $\mathrm{C}_{\mathrm{T}}$ values of sample MmiR-373, UmiR-373, and ActB were calculated using corresponding standard curves and then corrected to DNA amounts with ActB values. The sum of percentage of fully methylated (PMR) and unmethylated (PUR) DNA sample amounts (MmiR$373+$ UmiR-373 $=100 \%$ ) was calculated.

The primers used were: miR-373: M, 5'-ATTTTGG TTAATACGGTGAAATTTC-3', 5'-CTATCGCCCAAAC TAAAATACGAT-3'; U, 5'-TTTGGTTAATATGGTGAA ATTTTGT-3', 5'-CTCTATCACCCAAACTAAAATAC AAT-3'. ActB, 5'-TGGTGATGGAGGAGGTTTAGTA AGT-3', 5'-AACCAATAAAACCTACTCCTCCCTTAA-3'. ActG, 5'-TGGTGATGGAGGAGGCTCAGCAAGT-3', 5'-AGCCAATGGGACCTGCTCCTCCCTTGA-3'.

Chromatin immunoprecipitation (ChIP). ChIP was performed using ChIP-IT ${ }^{\mathrm{TM}}$ Express kit (Active Motif) using $2 \mu \mathrm{g}$ ChIPvalidated antibodies (Mecp2, MBD2 and mouse IgG, Active Motif; MBD1, Invitrogen). The presence was determined with primers to product a $201 \mathrm{bp}$ size (5'-AGATCGAGAC CATCCTGGCTAACA-3'; 5'-TGAGAATGAGTCTTGCT CTGTCGC-3'), the enrichment of RNA pol II at the GAPDH gene (116 bp size) was quantified as endogenous control. ChIP-qPCR reactions were performed using $\mathrm{SYBR}^{\circledR}{ }_{-}$ GreenER $^{\mathrm{TM}}$ Assay (Invitrogen) in a genomic DNA model. The protein enrichment was expressed as the ratio of immunoprecipitated DNA (IP-DNA) to the total amount of DNA in the chromatin sample (input). Fold change was calculated as the ratio of IP-DNA/input (target) to IPDNA/input (RNA PoI II) and then normalized to control.

Vectors. Full-length ORF-cDNA clone of human MBD2 designed as pDONR_MBD2 (Accession: NM_003927.2) was purchased from GeneCopoeia (USA). Small interfering RNA against MBD2 (MBD-siRNA, Accession: NM_003927) was purchased from Applied Biosystems (USA). Promoter luciferase reporter plasmid harboring the $\mathrm{CpG}$ island was constructed and was designated as pGL4-373-Prom following a previously described approach (33). In brief, miR-373 gene locus was screened in database (http://genome.ucsc.edu/), a $5-\mathrm{kb}$ fragment upstream by pre-miR-373 sequence was analyzed (http://www.fruitfly.org/seq_tools/promoter) to figure out putative promoter and transcriptional start site (TSS), and then CpG island was predicted with Methprimer software (http://www.urogene.org/methprimer/). As result, a 402 bases putative $\mathrm{CpG}$ island spanning from -251 to $+150 \mathrm{bp}$ containing $26 \mathrm{CpG}$ dinucleotides was found present in the 5'-flank region of human miR-373 gene (predicted TSS is recognized as +1$)$.

A 726 bp fragment of miR-373 gene (GenBank accession no. NR_029866) were amplified from QBC $_{939}$ cell genomic DNA by PCR using specific primers: 5'-CGATGGTACCTG GAAAGTGCTGCGACATTT-3' (sense), which contains an artificial KpnI site, and 5-TCATGCTAGCAGAGGTTGG CCTCCAATCAT-3' (antisense), which contains an artificial NheI site and four protective bases. The PCR-amplified fragments were digested with $\mathrm{KpnI} / \mathrm{HneI}$ and then inserted into pGL4.22-basic plasmid (Promega) to generate miR-373 gene promoter luciferase reporter plasmid designated as pGL4-373-Prom.
Establishment of stable cell line. pGL4-m373-prom or pGL4u373-prom was transfected into HEK293 cells within 35-mm dishes using Lipofectamine ${ }^{\mathrm{TM}}$ LTX and Plus Reagent (Invitrogen). At $24 \mathrm{~h}$ post-transfection, the cells were then divided into $100-\mathrm{mm}$ dishes at various cell densities and incubated in RPMI containing $2.0 \mu \mathrm{g} / \mathrm{ml}$ of puromycin for 7 days followed by maintained with dosage of $1.0 \mu \mathrm{g} / \mathrm{ml}$. The stable cell line established was designated as HEKm373-prom and HEK-u373-prom.

In vitro DNA methylation. pGL4-373-prom were methylated in vitro using M.SssI (4 U/ $\mu$ g DNA) (New England) in the presence of $160 \mu \mathrm{M} \mathrm{S}$-adenosylmethionine (SAM) for $16 \mathrm{~h}$. The methylated DNA redesigned as pGL4-m373-prom was digested with restriction enzyme either SalI which is blocked by M.SssI or BamI which is not blocked by M.SssI. Sensitivity to SalI and resistance to BamI indicates that the DNA was efficiently methylated.

Promoter luciferase reporter gene assay. Transfection was performed with Lipofectamine LTX (Invitrogen) in 24-well plate. Each transfection contains 500 ng/well pGL4-m373prom, pGL4-u373-prom or pGL4-Control vector and $100 \mathrm{ng} /$ well of pRL-TK. Cells were harvested at $72 \mathrm{~h}$ after transfection and lysates were analyzed for luciferase activity using the Dual Luciferase Reporter Assay (Promega).

siRNA knock-down study. Silencer ${ }^{\circledR}$ Select siRNAs against MBD2 and scrambled control siRNA (Applied Biosystems) was transfected using Silencer siRNA Transfection II Kit (Applied Biosystems). Each transfection contained $12.5 \mu 1$ of $1 \mu \mathrm{M}$ siRNA in a total $2500 \mu 1$ transfection mixture. Seventy-two hours post-transfection, the nearly confluent cells were used for RT-PCR and ChIP assay.

Western blotting. Proteins $(50 \mu \mathrm{g})$ were analyzed by Western blotting with primary antibody against Mecp2 (1:500, SigmaAldrich), MBD1 (1:1000, Millipore) and MBD2 (1:1000, Millipore), which produce a signal of $\sim 75, \sim 61$ and $\sim 50 \mathrm{kDa}$, respectively. GAPDH antibody (1:5000, abcam) was used as a loading control.

Statistical analysis. Student's t-test, one-way ANOVA and Pearson was used depending to data characteristic. Data are presented as mean \pm SEM. ${ }^{*} \mathrm{p}<0.05 ;{ }^{* *} \mathrm{p}<0.01$; p-values of $<0.05$ were considered to be significant. Survival duration was calculated via the Kaplan-Meier method. The log-rank test was employed for comparison of cumulative survival rate and disease-free survival in the patient group.

\section{Results}

Down-regulation of miR-373 is associated with poor cell differentiation, advanced clinical stage and shorter survival in patients with hilar cholangiocarcinoma. In patients with hilar cholangiocarcinoma, significant down-regulation of miR-373 was observed in $\mathrm{QBC}_{939}$ cells and 35 (72.92\%) tumors including 7 undetectable samples $(\mathrm{p}<0.01)$ (Fig. 1A). Fold-change analysis showed a 2.94-fold decrease in tumor group compared to control ( $\mathrm{p}<0.01$, Fig. 1B). 
A

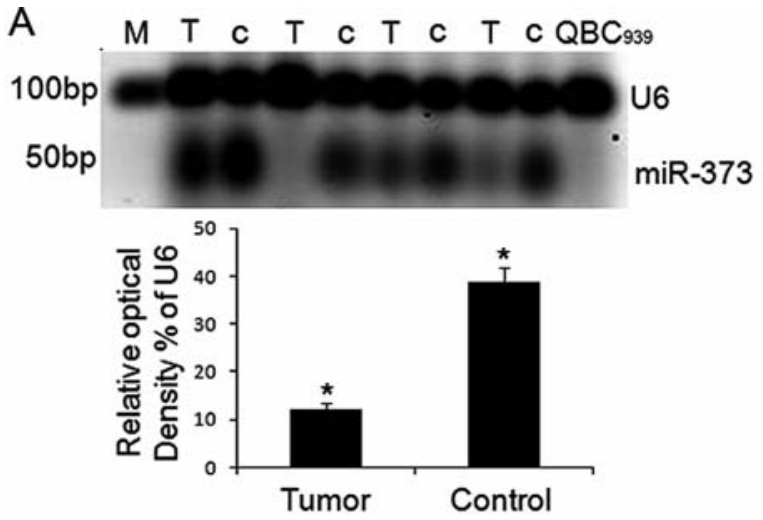

B
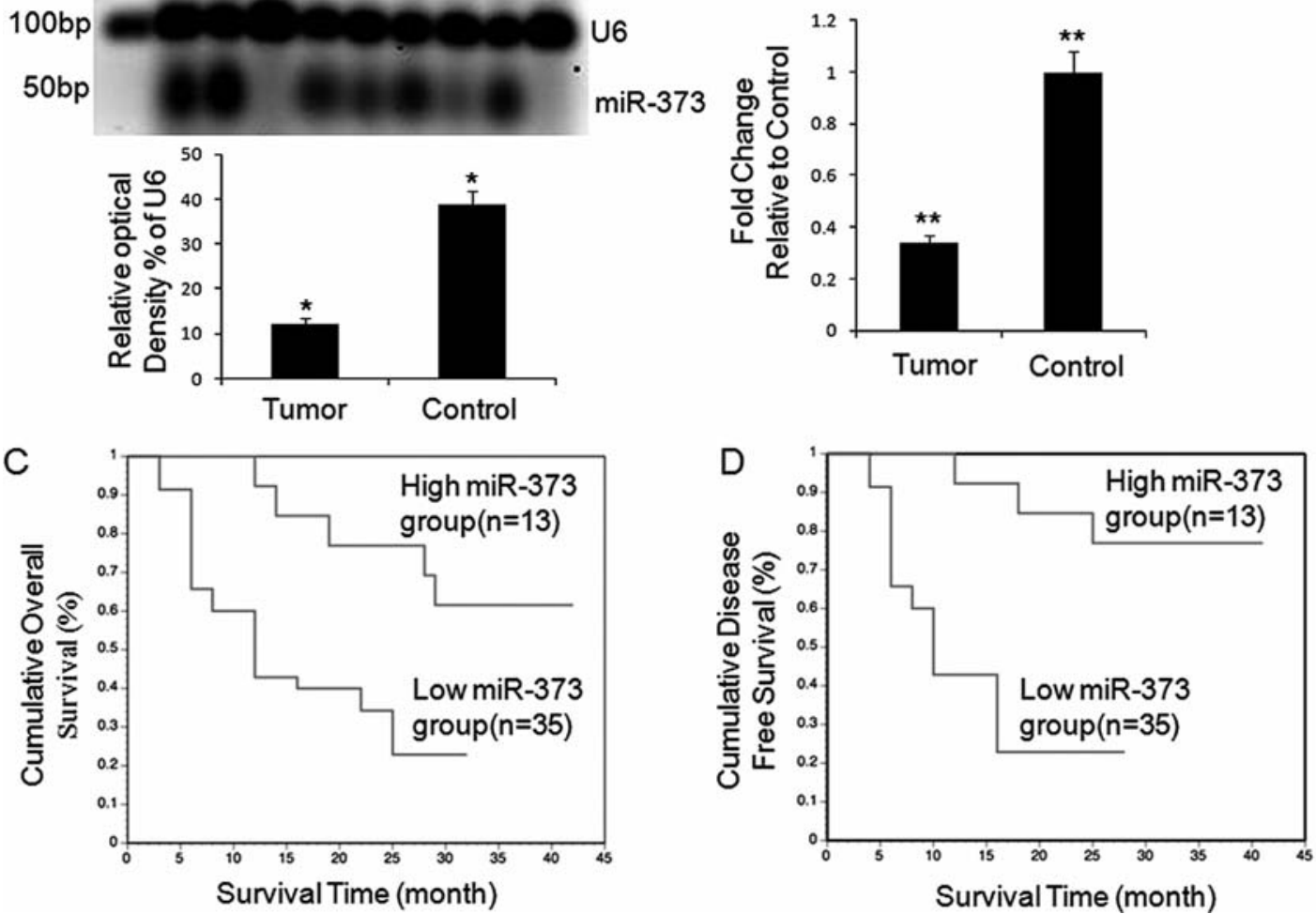

Figure 1. Expression of miR-373 and association with clinicopathological factors in patients with hilar cholangiocarcinoma. (A) Representative expression of miR-373 in tissues and QBC $_{939}$ cells detected by RT-PCR (top panel), relative optical density normalized to U6 showed in bottom bar chart in tumor and in $\mathrm{QBC}_{939} . \mathrm{M}, \mathrm{T}$ and $\mathrm{C}$ donate DNA marker, tumor and control, repectively. (B) TaqMan microRNA assay of miR-373, data are depicted as mean \pm SEM from three independent experiments in triplicate for each sample. (C) Relationship between miR-373 expression and overall survival in the patients with hilar cholangiocarcinoma. The median overall survival time was 16.3 months and 29.7 months in low- and high-miR-373 group, respectively. (D) KaplanMeier disease-free survival, the median disease-free survival time were 11.2 months, 23.4 months in low- and high-miR-373 group, respectively. ${ }^{*} \mathrm{p}<0.05$ and ${ }^{* *} \mathrm{p}<0.01$ when compared to control.

In identification of correlation between miR-373 expression and clinicopathological factors, miR-373 showed low expression in specimens with poor cell differentiation $(\mathrm{p}=0.031)$ and advanced clinical stages (stages III, IV vs. I, II) $(\mathrm{p}=0.017)$ (Table I) while no association was observed with age, gender, tumor size, different pathological type, Bismuth classification or lymphatic metastasis $(p>0.05)$. Further study was conducted to evaluate the miR-373 expression and survival. Kaplan-Meier analysis showed that down-regulation of miR-373 was relevant to decreased overall survival (Fig. 1C) and disease-free survival (Fig. 1D, $\mathrm{p}<0.05, \log$-rank test).

Promoter-associated CpG island of miR-373 is hypermethylated in $Q B C_{939}$ cells and hilar cholangiocarcinoma. According to definition of $\mathrm{CpG}$ island which is depicted as a region with at least $200 \mathrm{bp}$ and with a GC percentage that is greater than $50 \%$ and with an observed/expected $\mathrm{CpG}$ ratio that is greater than $60 \%$. A 402 base canonical $\mathrm{CpG}$ island encompassing a predicted transcription start site (TSS) was screened to present at the 5'-flank region of miR-373 gene (Fig. 2A). As seen in Fig. 3A, promoter luciferase reporter assay showed 73.2-fold relative luciferase activity of pGL4373-prom compared to pGL4-control vector which suggests that the predicted fragment behaves as a promoter to drive miR-373 gene expression.
Methylation status of promoter-associated CpG island was investigated with standard MSP and MethylSYBR techniques. In standard MSP, methylation presented in 38 $(38 / 48,79.17 \%)$ tumors including 26 homozygous methylation and 12 heterozygous methylation indicated by methylated band only, or both methylated and unmethylated bands, respectively. Heterozygous methylation was also observed in 5 control tissues $(\mathrm{p}<0.01$, Fig. $2 \mathrm{~B})$. This result was validated by following qMSP with fluorescent signal was detected in 38 cancer, 5 non-cancer samples. The value of PMR was 87.4 and $14.7 \%$ in tumor and control group, respectively (Fig. 2C, p<0.01).

To investigate the correlation between promoter methylation and miR-373 expression, we devided methylation into 4 groups according to the PMR values: suppermethylation (PMR above 90.00\%), hypermethylation (PMR ranges from 42.00 to $89.99 \%$ ), standard methylation (PMR ranges from 20.00 to $41.99 \%$ ), and hypomethylation (PMR belows $20.00 \%)$. Comparing with counterparts, miR-373 expression decreased distinctly in $88.5 \%$ (23/26) supermethylated samples $(\mathrm{p}<0.01)$, comparative reduction in 7 hypermethylated samples $(p<0.05)$ and dramatic increase was seen in 10 hypomethylated tumors and 43 control tissues $(p<0.01)$, no difference was detected in 5 standard methylated samples $(p>0.05)$. Interestingly, 3 suppermethylated tumors were characterized by a relatively high miR-373 expression (samples 14, 18 and 
A

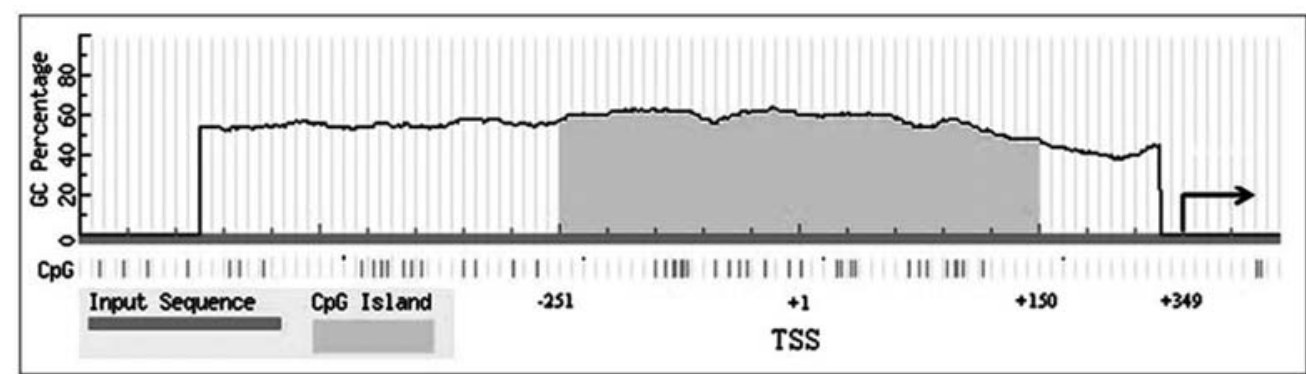

ctggggaagggaagggggcctcctgggetctcacctgacacaactaagggaatctgggttagatggtgaaagga agagaaggttcagagggcggctgtgCGcctgCGcCGggCGCGgCGgctcacacctgtaatcccagcaC GttgggaggcCGaggcaggCGgatcaCGaggtcaggagatCGagaccatcctggctaacaCGgtgaaa cccCGtctctactaaaaatacaaaaaatgagcCGggCGtggtggCGggCGcCtgtagtcccagctacttgg gaggctgaggcaggagaatggCGtgaaccCGggagg CGgagtttgcagtgagcCGagatCGCGccac CGcactccagcctgggCGacagagcaagactcattctc……GGGATACTCAAAATGGGGGCG CTTTCCTTTTTGTCTGTACTGGGAAGTGCTTCGATTTTGGGGTGTCCC

B
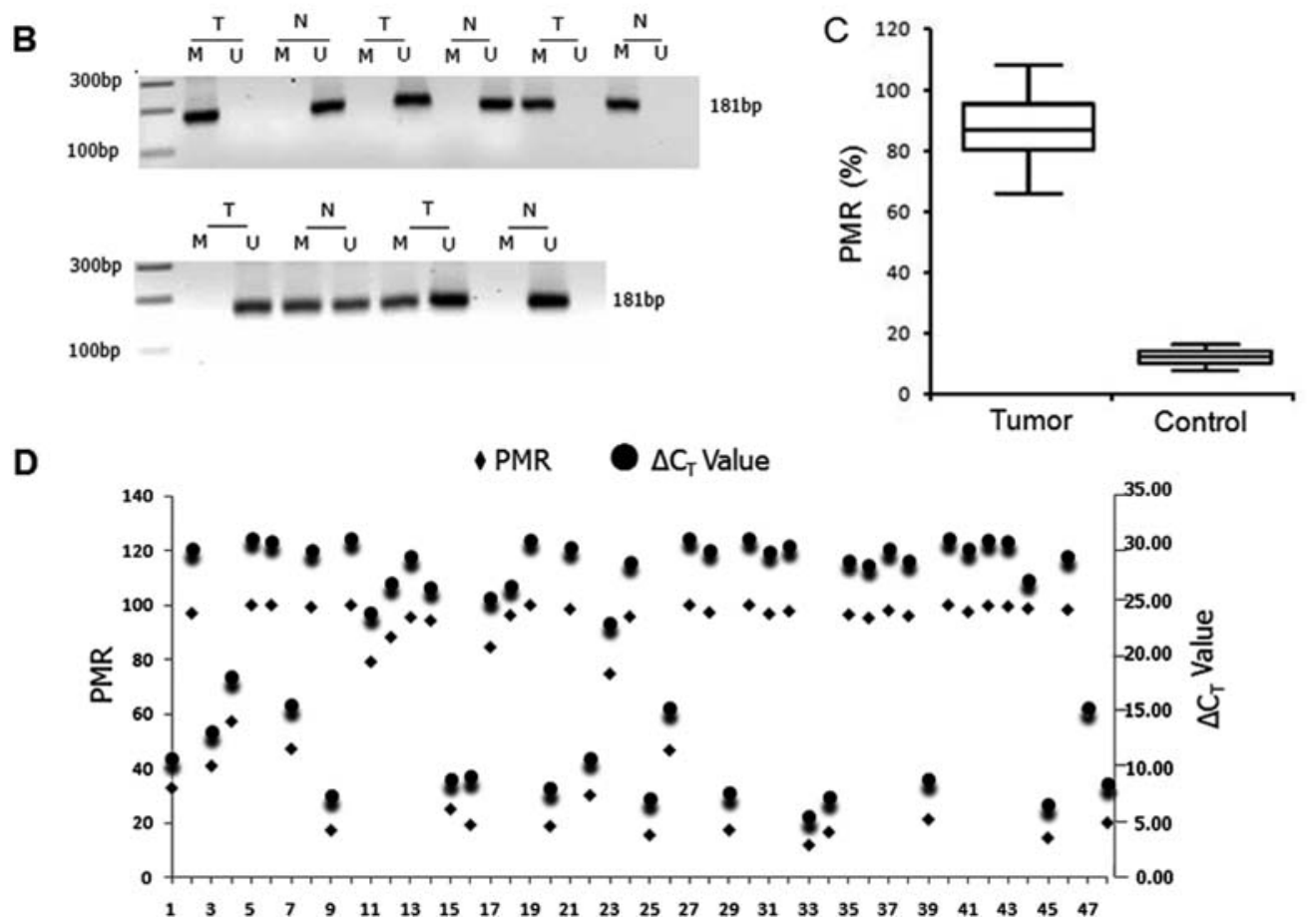

Figure 2. Methylation of miR-373 promoter-associated $\mathrm{CpG}$ island in hilar cholangiocarcinoma. (A) Top panel, schematic drawing of the putative CpG island. Bottom panel, sequence information of miR-373 gene including partial 5'-flank region. TSS in bold uppercase indicated by an arrow, CpG island is underlined and CpG dinucleotides are capitalized and italic, pre-miR-373 sequences are capitalized. (B) DNA methylation detected by MSP. Five representative cases show homozygous methylation (cases 1 and 3), heterozygous methylation (cases 4 and 5) and unmethylated (case 2), respectively. Lanes labeled M and U denote products amplified by primers recognizing methylated and unmethylated sequences. (C) PMR of miR-373 promoter-associated CpG island calculated from MethySYBR qMSP, data are depicted as mean \pm SEM from three independent experiments in triplicate for each sample, ${ }^{* *}$ p $<0.01$ when compared to control. (D) Relationship between CpG island methylation and miR-373 expression in hilar cholangiocarcinoma. PMR values were plotted at the lefthand scale. $\Delta \mathrm{C}_{\mathrm{T}}$ values relative to U6 were plotted at the right-hand scale for miR-373 expression (high $\Delta \mathrm{C}_{\mathrm{T}}$ value indicated low expression).

44). Despite these 3 extra tumors, promoter methylation demonstrated a contrary relationship with expression of miR-373 in hilar cholangiocarcinoma (Fig. 2D).

The promoter-associated $C p G$ island regulates $m i R-373$ expression through the methylation pathway. To explore the contribution of $\mathrm{CpG}$ island methylation to the expression of miR-373, pGL4-373-prom was methylated in vitro. As shown in Fig. 3A, the relative luciferase activity dramatically decreased in pGL4-m373-prom $(\mathrm{p}<0.01)$. On the contrary, in demethylation treated $\mathrm{QBC}_{939}$ cells, in which miR-373 gene was inhibited by heterozygous promoter methylation, a 3.1-fold and 3.4-fold increase of miR-373 in cells treated with 5-aza-CdR alone or combination with TSA were 


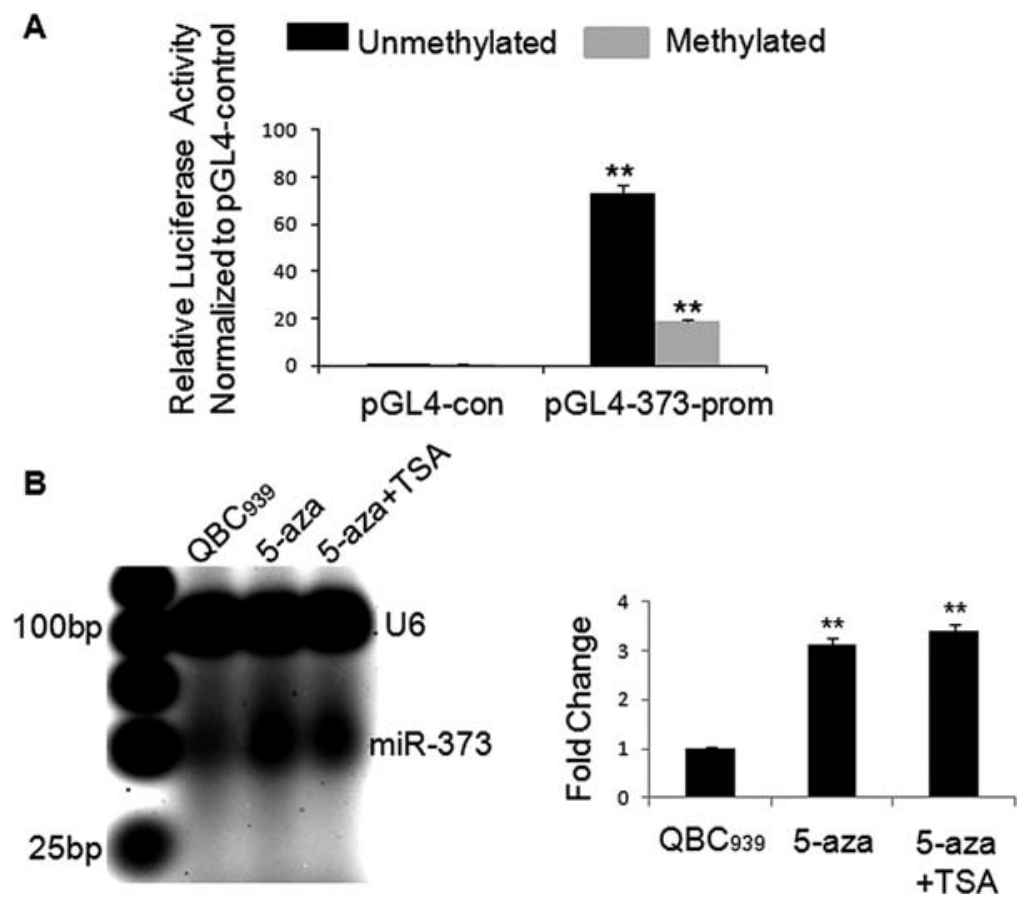

Figure 3. Methylation of $\mathrm{CpG}$ island regulates the expression of miR-373. (A) Promoter luciferase reporter gene assay for native and prior methylated miR-373 promoter. (B) miR-373 expression detected by TaqMan microRNA assay in $\mathrm{QBC}_{939}$ cells and treated by 5-aza-CdR alone or combination with TSA. Data are depicted as mean \pm SEM from three independent experiments in triplicate for each sample."* $<<0.01$ when compared to control.

detected (Fig. 3B). These results demonstrate that promoterassociated $\mathrm{CpG}$ island regulates miR-373 transcription through methylation pathway in hilar cholangiocarcinomas.

$M B D 2$ is recruited to the methylated $C p G$ island in hilar cholangiocarcinoma. Expression of Mecp2, MBD1 and MBD2 were explored at the level of protein with Western blotting. Compared to control, 2.9-fold increase of MBD2 was found in tumors while no difference of MBD1 and Mecp2 were detected (Fig. 4A).

Presence of Mecp2, MBD1 or MBD2 at CpG island region was assessed by $\mathrm{ChIP}$. In $\mathrm{QBC}_{939}$ cells and hilar cholangiocarcinoma, the $\mathrm{CpG}$ island fragment was markedly immunoprecipitated by MBD2. Fold enrichment analysis showed a 4.4-fold increase compared with control $(\mathrm{p}<0.01$, Fig. 4B, bottom panel). In contrast, anti-Mecp2 and MBD1 antibodies failed to precipitate of the DNA segment.

Fold enrichment of MBD2 in different methylation group was further analyzed. No significant difference was detected between supermethylation and hypermethylation or between standard and hypomethylation group ( $>>0.05)$, whereas remarkable difference was observed when comparing super-/ or hyper-methylation with standard-/or hypo-methylation group ( $\mathrm{p}<0.01$, Fig. $4 \mathrm{C}$ ). These findings suggest that MBD2 is selectively and specifically bound to the region of $\mathrm{CpG}$ island.

Promoter-associated $C p G$ island recruits MBD2 in pGL4m373-prom stable cell line. To unravel whether MBD2 is specifically recruited by methylated $\mathrm{CpG}$ island of miR-373 gene, exogenous MBD2 expression vector of $\mathrm{pDONR}^{\mathrm{TM}}$ MBD2 was transfected into stable cell line of HEK-u373prom or HEK-m373-prom. ChIP assay showed a 6.2-fold increase in pGL4-m373-prom compared to control, while no change was found in pGL4-u373-prom group (Fig. 5A, $\mathrm{p}<0.01)$.

$M B D 2$ knock-down up-regulates miR-373 in $Q B C_{939}$. In $\mathrm{QBC}_{939}$ cells with MBD2 knock-down by siRNA, a stimulation 4.3-fold increase of miR-373 expression is observed when compared with wild-type $\mathrm{QBC}_{939}$ cells (Fig. 5C). We then analyzed the specific binding of MBD2 to the region of $\mathrm{CpG}$ island. ChIP assay revealed that knock-down of MBD2 resulted in failure of recruitment of MBD2 on the miR-373 promoter-associated $\mathrm{CpG}$ island (Fig. 5B). Furthermore, the knock-down of MBD2 is not compensated by the binding of MBD1 or Mecp2. In addition, in MBD2 knock-down $\mathrm{QBC}_{939}$ cells, 5-aza-CdR and TSA treatments had an innocent effect on MBD2 binding (Fig. 5D).

\section{Discussion}

In released studies, miR-373 displays controversial characteristics in different cancers. In testicular germ cell tumor (34), esophageal (35) and breast cancer (36), miR-373 behaves as an oncogene. Whereas in prostate cancer (37) and malignant cholangiocytes (38), miR-373 shows characteristics of a tumor suppressor. Regardless of the divergence, miR-373 is well established to participate in tumorigenesis, invasion and metastasis by mediating gene expression. In this study, we present that miR-373 is dramatically down-regulated in hilar cholangiocarcinoma and correlated closely with poor cell differentiation, advanced clinical stage, and shorter survival. Our findings are in agreement with other reports $(37,38)$. How to explain these opposite results remains unclear, the expression patterns for individual miRNA with strict tissue- and clinical-feature- 
A
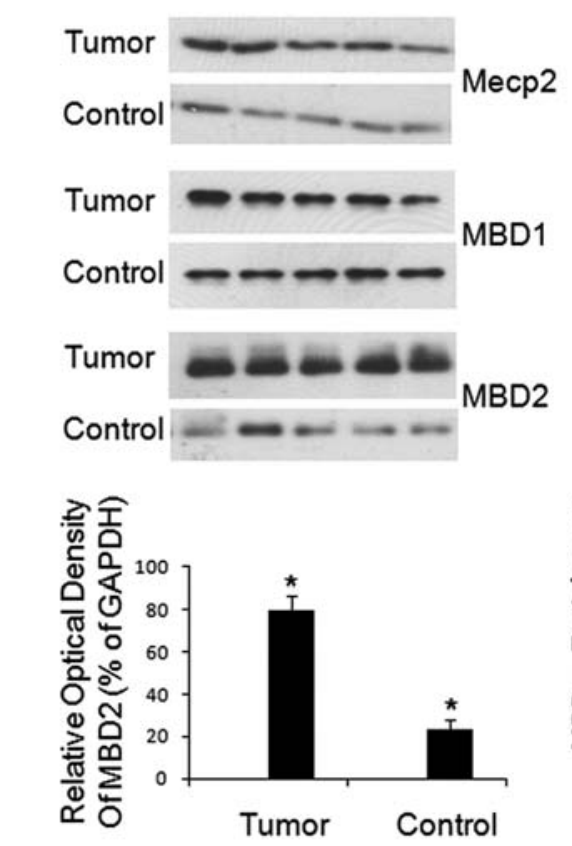

B
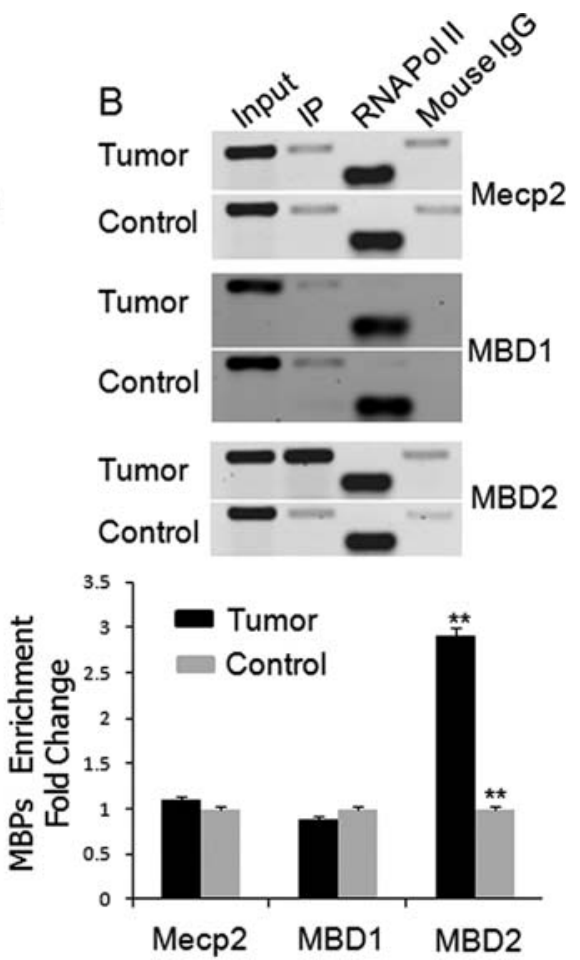

C

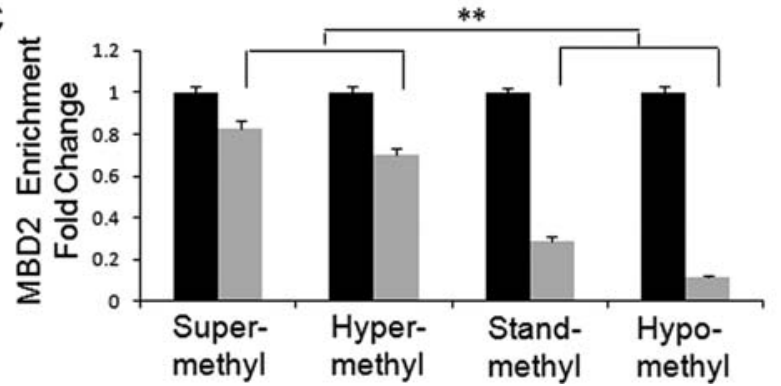

Figure 4. MBP expression and enrichment at promoter-associated $\mathrm{CpG}$ island of miR-373 in hilar cholangiocarcinoma. (A) Expression of Mecp2, MBD1 and MBD2 protein in hilar cholangiocarcinoma (top panel), relative optical density normalized to GAPDH of MBD2 showed in bottom bar chart. (B) ChIP analysis for enrichment of Mecp2, MBD1 and MBD2 at promoter-associated CpG island of miR-373. (C) Correlation between MBD2 enrichment and different frequency of $\mathrm{CpG}$ island methylation. Fold change is calculated as the ratio of IP-DNA/input (target) to IP-DNA/input (RNA poI II) and then normalized to control. Data obtained from three independent experiments in triplicate for each sample are depicted as mean \pm SEM, ${ }^{* *}<0.01$ when compared to control.

specificity, and different target gene involved in unique regulative network in various cancers could lead discrepant conclusions.

Methylation of promoter-associated $\mathrm{CpG}$ island is a frequent event in human cancer (39). Great effort has been devoted to understand the relevance of aberrant $\mathrm{CpG}$ methylation patterns and their roles in gene transcription $(40,41)$. The inverse correlation of miR-373 expression with progression in hilar cholangiocarcinoma prompted us to explore the molecular mechanism underlying miR-373 suppression. In the present study, miR-373 promoterassociated $\mathrm{CpG}$ island is found to be hypermethylated in tumor tissues and $\mathrm{QBC}_{939}$ cells. Reciprocal assay is performed with demethylation of the $\mathrm{CpG}$ island by treatment of $\mathrm{QBC}_{939}$ using 5-aza-CdR or combination with TSA, which contributes reactivation of miR-373. In addition, pre-methylation of pGL4-373-prom in vitro restrained the luciferase activity. To sum up, results presented here provide evidence that promoterassociated $\mathrm{CpG}$ island methylation is a major cause of miR373 gene suppression in hilar cholangiocarcinoma.
Methylation study requires precise identification of gene promoter region and $\mathrm{CpG}$ island. The complete sequence and function of miR-373 transcript has previously been validated (34). We predicted the promoter region of miR-373 and a 402 bp $\mathrm{CpG}$ island was found. The promoter activity is authenticated by luciferase reporter gene assay which demonstrates that this predicted fragment is capable of driving the expression of the reporter gene. Hence, this theoretical region can be considered a canonical promoter.

Promoter-associated CpG-methylation, along with MBPs and HDACs, has been identified as a major epigenetic event in loss of gene expression during tumor progression $(42,43)$. In this study, we further investigated the involved MBPs in methylation-mediated suppression of miR-373 in hilar cholangiocarcinoma. ChIP assay displays that MBD2 selectively binds to methylated $\mathrm{CpG}$ sequences. Moreover, silencing conferred by DNA methylation and MBD2 enrichment in $\mathrm{QBC}_{939}$ cells is relieved by treatment with 5-aza-CdR and TSA. These findings indicate that MBD2 plays an important role in recruiting transcription-repressive machinery to the 

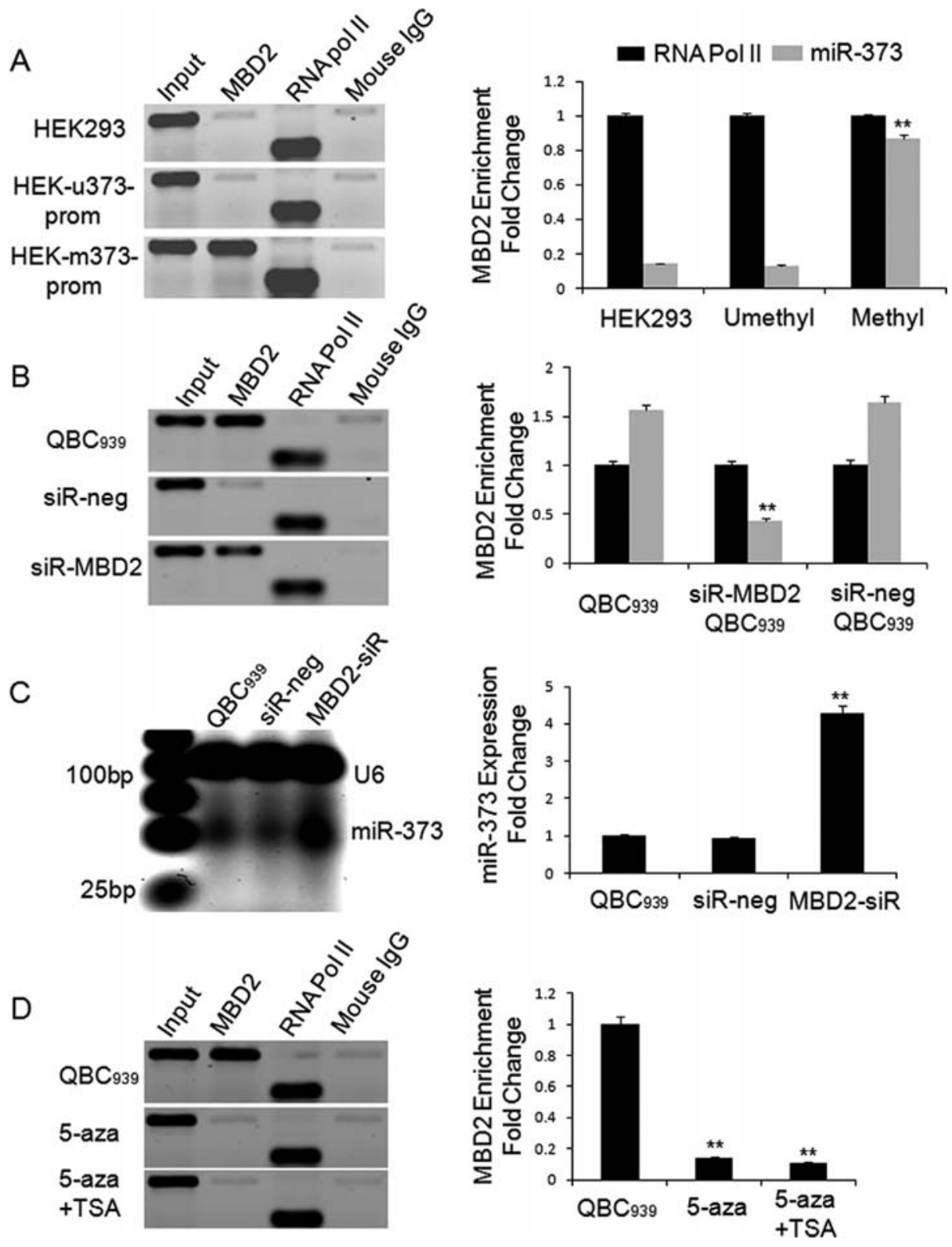

Figure 5. Enrichment of MBD2 at $\mathrm{CpG}$ island. (A) Enrichment of MBD2 in $\mathrm{CpG}$ island in HEK-m373-prom stable cells. (B) MBD2 enrichment in MBD2-siRNA QBC ${ }_{939}$ cells. (C) Knock-down of MBD2 induced an increase of miR-373 expression in QBC $_{939}$ cells detected by TaqMan microRNA assay. (D) Epigenetic treatment of $\mathrm{QBC}_{939}$ cell with 5-aza-CdR or combination with TSA eliminated the recruitment MBD2 at CpG island. Fold change is calculated as the ratio of IP-DNA/input (target) to IP-DNA/input (RNA poI II) and then normalized to control. Data obtained from three independent experiments in triplicate for each sample are depicted as mean $\pm \mathrm{SEM},{ }^{*} \mathrm{p}<0.05$ and ${ }^{* *} \mathrm{p}<0.01$ when compared to control.

methylated promoter, thereby suppressing the transcriptional activation of miR-373. Confirming these findings, siRNAdirected knock-down of MBD2 triggers a certain amount of stimulation of miR-373 in $\mathrm{QBC}_{939}$ cells. Taken together, these several lines of evidence suggest that MBD2 is an important factor which donates methylation-mediated inhibition of miR-373.

In this study, there are several interesting observations. First, heterozygous methylation of miR-373 indicated by both methylated and unmethylated bands in MSP is detected in 12 cancers and 5 normal bile duct tissues. It is contrary to 'all-or-none' manner of DNA methylation in regulating gene expression. Regarding mechanism is unclear, following reasons should be meaningful: i) miR-373 gene exhibits allele-specific DNA methylation (ASM) which means only one allele is methylated and the other one is unmethylated. ASM has been documented in numbers of cancer cases except for the imprinted regions and X chromosomes (44); ii) samples contain normal and malignant cells although multiple efforts have been adapted to obtain purified tissues; iii) miR-373 displays discrepant methylation in various differentiated cells. Secondly, in primary samples, three suppermethylated tumors were characterized to a relatively high miR-373 expression. The relevant mechanism underlying transcription that escapes from methylation-mediated suppression is unknown, but whether MBPs bind effectively to the methylated $\mathrm{CpG}$ dinucleotides may determine the expression level. 


\section{Acknowledgements}

This study is supported by Research Fund for the Doctoral Program of Higher Education of China (No. 20070487114) and Natural Science Foundation of Hubei Province, China; (No. 2008CBD159).

\section{References}

1. Klatskin G: Adenocarcinoma of the hepatic duct at its bifurcation within the portal hepatic. An unusual tumor with distinctive clinical and pathological features. Am J Med 38: 241-256, 1965

2. Martínez-González MN, Mondragón-Sánchez R, GómezGómez E, Núñez-Nateras RA, Bernal-Maldonado R Oñate-Ocaña LF and Ruiz-Molina JM: Epidemiologic factors and results of treatment in peripheral and perihilar cholangiocarcinoma. Rev Gastroenterol Mex 67: 250-258, 2002.

3. Hasegawa S, Ikai I, Fujii H, et al: Surgical resection of hilar cholangiocarcinoma: analysis of survival and postoperative complications. World J Surg 31: 1256-1263, 2007.

4. Nakeeb A, Tran KQ, Black MJ, et al: Improved survival in resected biliary malignancies. Surgery 132: 555-563, 2002

5. Paik WH, Park YS, Hwang JH, et al: Palliative treatment with self-expandable metallic stents in patients with advanced type III or IV hilar cholangiocarcinoma: a percutaneous versus endoscopic approach. Gastrointest Endosc 69: 55-62, 2009.

6. Heimbach JK, Gores GJ, Haddock MG, et al: Predictors of disease recurrence following neoadjuvant chemoradiotherapy and liver transplantation for unresectable perihilar cholangiocarcinoma. Transplantation 82: 1703-1707, 2006.

7. Nakeeb A and Pitt HA: Radiation therapy, chemotherapy and chemoradiation in hilar cholangiocarcinoma. HPB (Oxford) 7: 278-282, 2005

8. Aljiffry M, Walsh MJ and Molinari M: Advances in diagnosis, treatment and palliation of cholangiocarcinoma: 1990-2009. World J Gastroenterol 15: 4240-4262, 2009.

9. Klose RJ and Bird AP: Genomic DNA methylation: the mark and its mediators. Trends Biochem Sci 31: 89-97, 2006.

10. Watt F and Molloy PL: Cytosine methylation prevents binding to DNA of a HeLa cell transcription factor required for optimal expression of the adenovirus major late promoter. Genes 2 : 1136-1143, 1988

11. Bird AP and Wolffe AP: Methylation-induced repression - belts, braces, and chromatin. Cell 99: 451-454, 1999.

12. Buschhausen G, Wittig B, Graessmann M and Graessmann A: Chromatin structure is required to block transcription of the methylated herpes simplex virus thymidine kinase gene. Proc Natl Acad Sci USA 84: 1177-1181, 1987.

13. Wade PA: Methyl CpG binding proteins: coupling chromatin architecture to gene regulation. Oncogene 20: 3166-3173, 2001.

14. Delaval K and Feil R: Epigenetic regulation of mammalian genomic imprinting. Curr Opin Genet Dev 14: 188-195, 2004.

15. Weaving LS, Williamson SL, Bennetts B, et al: Effects of MECP2 mutation type, location and X-inactivation in modulating Rett syndrome phenotype. Am J Med Genet 118A: 103-114, 2003.

16. Cedar $\mathrm{H}$ and Bergman $\mathrm{Y}$ : Linking DNA methylation and histone modification: patterns and paradigms. Nat Rev Genet 10: 295-304, 2009.

17. Hendrich B, Hardeland U, Ng HH, Jiricny J and Bird A: The thymine glycosylase MBD4 can bind to the product of deamination at methylated CpG sites. Nature 401: 301-304, 1999.

18. Nan X, Campoy FJ and Bird A: MecP2 is a transcriptional repressor with abundant binding sites in genomic chromatin. Cell 88: 471-481, 1997.

19. Fujita N, Takebayashi S, Okumura K, Kudo S, Chiba T, Saya H and Nakao M: Methylation-mediated transcriptional silencing in euchromatin by methyl-CpG binding protein MBD1 isoforms. Mol Cell Biol 19: 6415-6426, 1999.

20. $\mathrm{Ng} \mathrm{HH}$, Zhang Y, Hendrich $\mathrm{B}$, et al: MBD2 is a transcriptional repressor belonging to the MeCP1 histone deacetylase complex. Nat Genet 23: 58-61, 1999.

21. Saito $\mathrm{M}$ and Ishikawa F: The $\mathrm{mCpG}$-binding domain of human MBD3 does not bind to $\mathrm{mCpG}$ but interacts with NuRD/Mi2 components HDAC1 and MTA2. J Biol Chem 277: 35434-35439, 2002 .
22. Birney E, Stamatoyannopoulos JA, Dutta A, et al: Identification and analysis of functional elements in $1 \%$ of the human genome by the ENCODE pilot project. Nature 447: 799-816, 2007.

23. Lecellier $\mathrm{CH}$, Dunoyer P, Arar K, et al: A cellular microRNA mediates antiviral defense in human cells. Science 308: 557-560, 2005.

24. Tomaru Y and Hayashizaki Y: Cancer research with non-coding RNA. Cancer Sci 97: 1285-1290, 2006.

25. Calin GA and Croce CM: MicroRNA signatures in human cancers. Nat Rev Cancer 6: 857-866, 2006.

26. Lee Y, Kim M, Han J, Yeom KH, Lee S, Baek SH and Kim VN: MicroRNA genes are transcribed by RNA polymerase II. EMBO J 23: 4051-4060, 2004.

27. Guil S and Esteller M: Mechanisms of disease: methyl-binding domain proteins as potential therapeutic targets in cancer. Int J Biochem Cell Biol 41: 87-95, 2009.

28. Li D, Chen J, Gao Z, Li X, Yan X, Xiong Y and Wang S: 67-kDa laminin receptor in human bile duct carcinoma. Eur Surg Res 42: 168-173, 2009.

29. Tang QB, Sun HW and Zou SQ: Effects of 5-aza-2-deoxycytidine on the growth and apoptosis of bile duct cancer cell line. J Huazhong Univ Sci Tech (Health Sci) 33: 34-36, 2004.

30. Livak KJ and Schmittgen TD: Analysis of relative gene expression data using real-time quantitative PCR and the 2(-Delta Delta C(T)) method. Methods 25: 402-408, 2001.

31. Hattermann K, Maximilian HM, Mentlein R, Schultka S and Held-Feindt J: A methylation-specific and SYBR-greenbased quantitative polymerase chain reaction technique for $\mathrm{O}^{6}$ methylguanine DNA methyltransferase promoter methylation analysis. Anal Biochem 377: 62-71, 2008.

32. Lo PK, Watanabe H, Cheng PC, et al: MethySYBR, a novel quantitative PCR assay for the dual analysis of DNA methylation and $\mathrm{CpG}$ methylation density. J Mol Diagn 11: 400-414, 2009

33. Changzheng C, Ling L and Harvey FL: MicroRNAs modulate hematopoietic lineage differentiation. Science 303: 83-86, 2004.

34. Voorhoeve PM, le Sage C, Schrier M, et al: A genetic screen implicates miRNA-372 and miRNA-373 as oncogenes in testicular germ cell tumors. Cell 124: 1169-1181, 2006.

35. Lee KH, Goan YG, Hsiao M, et al: MicroRNA-373 (miR-373) post-transcriptionally regulates large tumor suppressor, homolog 2 (LATS2) and stimulates proliferation in human esophageal cancer. Exp Cell Res 315: 2529-2538, 2009.

36. Huang Q, Gumireddy K, Schrier M, et al: The microRNAs miR-373 and miR-520c promote tumour invasion and metastasis. Nat Cell Biol 10: 202-210, 2008.

37. Yang K, Handorean AM and Iczkowski KA: MicroRNAs 373 and $520 \mathrm{c}$ are downregulated in prostate cancer, suppress CD44 translation and enhance invasion of prostate cancer cells in vitro. Int J Clin Exp Pathol 2: 361-369, 2009.

38. Meng F, Henson R, Lang M, et al: Involvement of human micro-RNA in growth and response to chemotherapy in human cholangiocarcinoma cell lines. Gastroenterology 130: 2113-2129, 2006.

39. Toyota M, Suzuki H, Yamashita T, Hirata K, Imai K, Tokino T and Shinomura Y: Cancer epigenomics: implications of DNA methylation in personalized cancer therapy. Cancer Sci 100: 787-791, 2009.

40. Esteller M and Almouzni G: How epigenetics integrates nuclear functions. Workshop on epigenetics and chromatin: transcriptional regulation and beyond. EMBO Rep 6: 624-628, 2005.

41. Lodygin D, Epanchintsev A, Menssen A, Diebold J and Hermeking H: Functional epigenomics identifies genes frequently silenced in prostate cancer. Cancer Res 65: 4218-4227, 2005.

42. Fraga MF and Esteller M: Towards the human cancer epigenome: a first draft of histone modifications. Cell Cycle 4: 1377-1381, 2005.

43. Sharma D, Blum J, Yang X, Beaulieu N, Macleod AR and Davidson NE: Release of methyl $\mathrm{CpG}$ binding proteins and histone deacetylase 1 from the Estrogen receptor alpha (ER) promoter upon reactivation in ER-negative human breast cancer cells. Mol Endocrinol 19: 1740-1751, 2005.

44. Shoemaker R, Deng J, Wang W and Zhang K: Allele-specific methylation is prevalent and is contributed by CpG-SNPs in the human genome. Genome Res 20: 883-889, 2010. 